\title{
Atitudes face à doação de gâmetas e gestação de substituição
}

\section{Attitudes toward gamete donation and surrogacy}

\author{
Nair Carolino (1) \\ Ana Galhardo (1,2) \\ Marina Cunha $(1,2$ \\ (1) Instituto Superior Miguel Torga, Portugal \\ (2) Centro de Investigação em Neuropsicologia e Intervenção Cognitivo-Comportamental, Portugal \\ Recebido: 18/02/2019; Revisto: 28/02/2019; Aceite: 28/02/2019.
}

https://doi.org/10.31211/rpics.2019.5.1.119

\section{Resumo}

Objetivo: A parentalidade constitui-se como um desejo comum a muitos indivíduos, mas em alguns casos a sua concretização implica o recurso a técnicas de reprodução medicamente assistida, como a doação de gâmetas ou a gestação de substituição. Em virtude da escassez de estudos sobre atitudes face à doação/receção de gâmetas e gestação de substituição, este estudo pretendeu explorar as atitudes de indivíduos em idade reprodutiva relativamente a estas técnicas. Métodos: Participaram 551 sujeitos com idades entre os 18 e os 40 anos, recrutados através de amostragem por bola de neve. Foi solicitado o preenchimento de um questionário desenvolvido especificamente para o estudo, disponibilizado numa plataforma online, que avaliou o posicionamento dos sujeitos face à doação/receção de gâmetas e gestação de substituição. Resultados: A maioria dos participantes revelou uma atitude positiva perante a doação/receção de gâmetas. No caso da doação a principal motivação indicada foi a de ajudar um casal que não pode ter filhos. Relativamente à receção de gâmetas, os dados sugerem tratar-se de uma circunstância bem aceite pelos participantes. Já no que se refere à gestação de substituição, ainda que exista um posicionamento favorável à sua legalização, nem todos os participantes considerariam essa possibilidade, ainda que aqueles que a equacionariam refiram que se sentiriam felizes por concretizar o sonho de se tornar mãe/pai. Conclusões: Na globalidade, a receção/doação de gâmetas é vista de um modo favorável. Aspetos como realizar o desejo de parentalidade e poder cuidar de uma criança desde o seu nascimento são relevantes, sugerindo uma menor valorização da componente genética. Por sua vez a doação de gâmetas parece relacionar-se com motivações altruístas, podendo ser potenciada com a existência de aconselhamento. A complexidade da gestação de substituição poderá contribuir para a existência de uma menor abertura, ainda que os sujeitos estejam de acordo com a sua legalização em Portugal.

Palavras-Chave: Obtenção de tecidos e órgãos; Gestação de substituição; Conhecimentos, atitudes e prática em saúde.

\section{DI\&D | ISMT}

rpics@ismt.pt https://rpics.ismt.pt
Publicação em Acesso Aberto (02019. O(s) Autor(es). Este é um artigo de acesso aberto distribuído sob a Licença Creative Commons Attribution, que permite uso, distribuição e reprodução sem restrições em qualquer meio, desde que o trabalho original seja devidamente citado.
Nair Carolino

Largo de Celas,

3000-132, Coimbra, Portugal

e-mail: naircarolino@hotmail.com 


\begin{abstract}
Aims: Parenting is a common desire for many individuals, although for some becoming a parent implies the use of assisted reproductive technologies, such as gamete donation or gestational surrogacy. Given the paucity of studies addressing attitudes towards gamete donation/reception and gestational surrogacy, the current study aimed to explore the attitudes of individuals of reproductive age concerning these techniques. Methods: The study was conducted in a sample of 551 subjects, aged between 18 and 40 years old. Participants were recruited through a snow ball sampling and completed an online questionnaire specifically developed for the study. The questionnaire encompassed a set of questions related the subjects' positioning towards gamete donation/reception and gestational surrogacy. Results: The majority of participants revealed a positive attitude towards the gamete donation/reception. The primary motivation for gamete donation was to help a couple who cannot have children. Concerning gamete reception, data suggest that this is a well-accepted circumstance by the participants. Regarding gestational surrogacy, although there is an approving position to its legalization, not all the participants would consider this possibility. Those who would consider it stated that they would feel happy to accomplish the dream of becoming a mother/father. Conclusions: Overall, gamete reception/donation is wellaccepted. Aspects such as achieve parenthood and being able to care for a child from birth are relevant, suggesting the genetic component to be less important. In turn, gamete donation seems to be related to altruistic motivations, and can be enhanced by the existence of counselling. The gestational surrogacy complexity may contribute to less openness, even if the subjects agree with its legalization in Portugal.
\end{abstract}

Keywords: Tissue and organ procurement; Gestational surrogacy; Health knowledge, attitudes, and practice.

\title{
Introdução
}

A parentalidade constitui-se como um desejo ou objetivo comum a muitos indivíduos/casais. Porém, nem todos os sujeitos conseguem alcançar uma gravidez de forma espontânea tendo, cada vez mais, que recorrer a técnicas médicas para concretizar o seu desejo (Boivin, Bunting, Collins, \& Nygren, 2007). Na atualidade o adiamento da parentalidade, principalmente nas mulheres, resulta de múltiplas alterações sociais, entre as quais uma maior aposta no prosseguimento da formação académica e valorização da carreira profissional (Lampic, Svanberg, Karlström, \& Tydén, 2006; Nouri et al., 2014). Neste sentido, e maioritariamente no Ocidente, o projeto da parentalidade tende a ser adiado para idades em que a probabilidade de ocorrência de infertilidade aumenta exponencialmente (Lampic et al., 2006; Rovei et al., 2010).

De acordo com o International Committee for Monitoring Assisted Reproductive Technology, a infertilidade pode ser definida como uma doença do sistema reprodutivo que se traduz na incapacidade de alcançar uma gravidez clínica após 12 meses de relações sexuais desprotegidas ou devido a danos na capacidade do sujeito para se reproduzir, quer como indivíduo, quer com o seu/sua parceiro/a (Zegers-Hochschild et al., 2017). Para além da definição médica, alguns autores referem que a infertilidade pode igualmente ser conceptualizada como uma condição social na qual os indivíduos ou casais evidenciam dificuldades em alcançar a parentalidade ou o papel reprodutivo na sociedade (Allan \& Mounce, 2015). Do ponto de vista da prevalência, estima-se que aproximadamente $9 \%$ dos casais em idade reprodutiva apresentem problemas de fertilidade (Boivin et al., 2007). Especificamente em Portugal, no estudo Afrodite (Silva-Carvalho \& Santos, 2009) foi encontrada uma prevalência ao longo da vida entre os $9 \%$ e os $10 \%$.

Os avanços tecnológicos no domínio da medicina da reprodução têm vindo a possibilitar a crescente resolução de problemas de fertilidade. De notar que o $18 .^{\circ}$ Relatório da European Society for 
Reproductive Medicine and Embriology aponta para uma expansão gradual, quer no número de tratamentos de reprodução medicamente assistida realizados na Europa, quer na maior variabilidade das modalidades de tratamento, conduzindo a um maior contributo para a taxa de natalidade dos diferentes países (De Geyter et al., 2018). Neste contexto, o alargamento das técnicas de reprodução medicamente assistida, ao englobar, por exemplo, o recurso a gâmetas/embriões doados, a gestação de substituição (GS) e a preservação de gâmetas, veio permitir dar resposta a situações complexas para as quais anteriormente não existiam opções disponíveis. Com efeito, a construção de família, a gravidez e a conceção sofreram alterações muito relevantes neste último século. Exemplo disso é o aumento significativo do número de nascimentos como resultado da doação de gâmetas, que nos Estados Unidos da América foi de 30.000 para 60.000 nos últimos 20 anos (Nahata, Stanley, \& Quinn, 2017). Na Europa, em 2014, o número de ciclos de tratamento realizados com ovócitos doados foi de 56 516, sendo 493 destes em Portugal (De Geyter et al., 2018). Face ao adiamento da parentalidade anteriormente mencionado, o recurso a gâmetas de dador, mais especificamente de ovócitos, constitui-se como um procedimento indicado para muitas mulheres que adiam a maternidade. No entanto, continua a ser uma questão sensível em virtude de desafiar a filiação genética da família, sendo esta valorizada por muitas sociedades (ESHRE Task Force on Ethics and Law, 2002). Paralelamente, aspetos relacionados com o anonimato dos dadores, a revelação do modo de conceção às crianças que resultam de tratamentos realizados com gâmetas doados e o designado "cross-border reproductive care" assumem-se como elementos que suscitam discussão (e.g., Pennings, Ravel, Girard, Domin-Bernhard, \& Provoost, 2018; Provoost, Van Rompuy, \& Pennings, 2018; Salama et al., 2018).

Um outro tópico controverso é o da GS, tendo esta diferentes enquadramentos legais consoante os países, sendo em muitos deles, proibida. Esta é uma técnica que apresenta múltiplos requisitos que a tornam um processo complexo ao envolver aspetos médicos, psicológicos e legais (Goldfarb et al., 2000). A GS é realizada com gâmetas do casal beneficiário ou gâmetas doados, sendo que os ovócitos não poderão ser doados pela gestante, ou seja, esta não possui vínculo genético com o embrião. $\mathrm{Na}$ generalidade, podem usar esta técnica, pessoas com ausência de útero ou condição médica relacionada com este órgão ou outro, que tornem uma gravidez claramente desaconselhada (Dar et al., 2015). No nosso país, até há bem pouco tempo, a GS não constava do Decreto-Lei n. ${ }^{\circ} 32 / 2006$, artigo 2. ${ }^{\circ}$, enquanto técnica de procriação medicamente assistida. Com a Lei n. ${ }^{\circ}$ 25/2016, de 22 de agosto, artigo $1 .^{\circ}$, passou a ser permitido "o acesso à gestação de substituição nos casos de ausência de útero, de lesão ou de doença deste órgão que impeça de forma absoluta e definitiva a gravidez". Ainda assim, existem detalhes da lei acerca dos quais se aguarda reformulação face ao Acórdão do Tribunal Constitucional n. ${ }^{\circ}$ 225/2018.

Atendendo à escassa informação em Portugal acerca das atitudes da população em geral relativas ao recurso a técnicas de reprodução medicamente assistida com gâmetas de dador e à GS, a presente investigação procurou explorar aspetos relativos às atitudes de indivíduos em idade reprodutiva no que respeita a estas técnicas. $O$ conhecimento destes elementos poderá contribuir para o delinear de medidas de sensibilização potenciadoras de atitudes mais informadas acerca da doação/receção de gâmetas e da gestação de substituição. 


\section{Método}

\section{Participantes}

Para a participação no estudo foi estipulado como critério de inclusão os sujeitos encontrarem-se em idade reprodutiva, definida entre os 18 e os 40 anos de idade. Participaram 551 indivíduos (com idades compreendidas entre os 18 e os 40 anos, inclusive), de ambos os sexos. A Tabela 1 apresenta as características sociodemográficas dos participantes. Da amostra recolhida, 432 (78,4\%) sujeitos são do sexo feminino e 119 (21,6\%) do sexo masculino, com uma média de idade de 27,56 anos $(D P=5,05)$. Considerando o estado civil, 75,0\% ( $n=413$ ) dos sujeitos são solteiros(as), $23,6 \%$ ( $n=130$ ) são casados(as) e $1,4 \%(n=8)$ são divorciados(as)/viúvos(as). A maioria dos participantes não tem filhos (97,8\%). Relativamente às habilitações literárias, $40,1 \%$ dos sujeitos detêm o grau de mestre e $37,4 \%$ o grau de licenciatura, apresentando, em média 15,58 anos de escolaridade.

\section{Tabela 1}

\section{Características Sociodemográficas}

\begin{tabular}{|c|c|c|c|}
\hline \multirow[b]{2}{*}{ Sexo } & \multicolumn{3}{|c|}{ Amostra Total } \\
\hline & & $N$ & $\%$ \\
\hline Masculino & & 119 & 21,6 \\
\hline \multirow[t]{3}{*}{ Feminino } & & 432 & 78,4 \\
\hline & Total & 551 & 100 \\
\hline & & $M(D P)$ & Variação \\
\hline Idade & & $27,56(5,05)$ & $18-40$ \\
\hline Anos de Escolaridade & & $15,58(2,02)$ & $9-22$ \\
\hline Estado Civil & & $N$ & $\%$ \\
\hline Solteiro/a & & 413 & 75,0 \\
\hline Casado/a & & 130 & 23,6 \\
\hline \multirow[t]{2}{*}{ Divorciado/a ou Viúvo/a } & & 8 & 1,4 \\
\hline & Total & 551 & 100 \\
\hline Filhos & & $N$ & $\%$ \\
\hline Tem & & 12 & 2,2 \\
\hline \multirow[t]{2}{*}{ Não tem } & & 539 & 97,8 \\
\hline & Total & 551 & 100 \\
\hline Habilitações Académicas & & $N$ & $\%$ \\
\hline Até ao Ensino Secundário/Equivalente & & 92 & 16,6 \\
\hline Licenciatura & & 206 & 37,4 \\
\hline Mestrado & & 221 & 40,1 \\
\hline Doutoramento & & 14 & 2,6 \\
\hline Pós-Graduação & & 4 & 0,7 \\
\hline \multirow[t]{2}{*}{ Não responderam } & & 14 & 2,5 \\
\hline & Total & 551 & 100 \\
\hline
\end{tabular}




\section{Instrumentos}

Questionário Sociodemográfico. Os dados sociodemográficos recolhidos englobaram questões relativas ao sexo, idade, estado civil e habilitações académicas. Foi ainda recolhida informação relativamente ao facto de os participantes terem ou não filhos.

Em virtude da inexistência de instrumentos específicos para avaliação das atitudes face à doação de gâmetas e à gestação de substituição, foi desenvolvido um conjunto de questões em função dos objetivos estipulados para o presente estudo, as quais resultaram da revisão da literatura e discussão entre os investigadores:

Questionário de Atitudes face à Doação de Gâmetas. Este questionário integrou questões sobre a atitude do sujeito perante a possibilidade de um amigo ser dador/recetor de gâmetas e em que medida esta técnica é um recurso relevante para as pessoas com infertilidade. Foi também colocada a questão sobre a hipótese de se tornar dador/recetor de gâmetas e como se sentiria face a essa possibilidade, bem como recolhida informação relativamente ao facto de o sujeito ser ou ter sido dador de órgãos ou tecidos. Assim, e a título de exemplo, foram elaboradas as seguintes questões: "Colocaria a hipótese de alguma vez doar gâmetas (ovócitos/espermatozoides)?", "Como se sentiria face à possibilidade de ter que recorrer a gâmetas de dador(es)?" [Atitudes face à possibilidade de ter que recorrer a gâmetas de dador(es)], "É ou já foi dador de órgãos ou tecidos (ex., sangue, medula, óvulos)?’. Neste questionário, e consoante o tipo de questão, as respostas são assinaladas num formato dicotómico (Sim/Não) ou numa escala de 5 pontos (variando de 1 = Discordo totalmente a $5=$ Concordo totalmente ou $1=$ Nada verdadeiro para mim a $5=$ Totalmente verdadeiro para mim).

Questionário de Atitudes face à Gestação de Substituição. O presente questionário recolheu a opinião dos sujeitos em relação à legalização da GS, as suas atitudes face a um amigo/a que pretendesse recorrer a esta técnica ou uma amiga que pretendesse atuar como gestante de substituição e as atitudes face à possibilidade de os respondentes virem a fazer uso desta técnica, em caso de necessidade. Neste questionário foram inseridas questões, como por exemplo: "Concorda com a legalização da gestação de substituição, também conhecida por maternidade de substituição ou "barriga de aluguer", no caso de mulheres com ausência de útero ou situação médica impeditiva de ocorrência de uma gravidez?" e "Se não conseguisse ter filhos de forma espontânea e querendo ter um filho biológico, consideraria a hipótese de recorrer à gestação de substituição?'. Uma vez mais neste questionário, e consoante o tipo de questão, as respostas são assinaladas num formato dicotómico (Sim/Não) ou numa escala de 5 pontos (indo de 1 = Discordo totalmente a $5=$ Concordo totalmente; ou $1=$ Nada verdadeiro para mim a $5=$ Totalmente verdadeiro para mim). 


\section{Procedimentos}

$\mathrm{Na}$ sequência da elaboração dos instrumentos anteriormente descritos, procedeu-se à sua disponibilização na plataforma online LimeSurvey, entre fevereiro e abril de 2015. Trata-se de uma amostra por conveniência, recolhida com recurso ao método de amostragem bola de neve. Num primeiro momento, foi criada uma mailing-list com os contactos de um grupo de indivíduos da comunidade, que foram convidados, via correio eletrónico, a participar no estudo e a colaborar na sua divulgação. O e-mail enviado disponibilizava informações acerca do âmbito e objetivos da investigação e o link de acesso à plataforma na qual o protocolo era preenchido. Após o acesso ao referido link, era solicitado o consentimento informado aos participantes, sem o qual não poderiam prosseguir. As questões foram assinaladas como de resposta obrigatória, pelo que não se verificaram valores em falta.

\section{Análise Estatística}

A análise estatística dos dados foi realizada com recurso ao software Statistical Package for the Social Sciences (SPSS), versão 20 (IBM SPSS Statistics). Conduziram-se testes paramétricos em virtude do número de sujeitos da amostra (Marôco, 2010).

Para a descrição da amostra, realizaram-se análises descritivas, tendo sido obtidas as frequências e percentagens para as variáveis categóricas, tais como, sexo, estado civil e habilitações académicas. Para as variáveis contínuas, como a idade, foram calculadas médias e desvios-padrão. Para a análise das atitudes face à doação de gâmetas e à gestação de substituição, recorreu-se à análise das frequências e percentagens das respostas dos participantes. A existência de eventuais diferenças entre sexos foi analisada com recurso ao teste do Qui-quadrado.

Em todas as análises realizadas considerou-se o intervalo de confiança de $95 \%$.

\section{Resultados}

\section{Atitudes face à doação de gâmetas}

A maioria dos participantes $(70,9 \% ; n=370)$ do estudo não é dador, quer de órgãos, quer de tecidos. No que respeita às atitudes perante a doação de gâmetas, 52,0\% dos indivíduos apoiariam totalmente um/a amigo/a que quisesse doar gâmetas a outro casal e 53,0\% apoiaria totalmente se um/a amigo/a tencionasse ser recetor de gâmetas. Face à afirmação "se uma pessoa não pode ter filhos, então não deveria tê-los", 82,1\% $(n=428)$ dos indivíduos discordam totalmente e 48,0\% $(n=250)$ concordam totalmente que a doação de gâmetas é uma boa técnica para ajudar casais que não podem ter filhos de uma forma espontânea (Tabela 2). 


\section{Tabela 2}

Atitudes Face à Doação de Gâmetas

\begin{tabular}{|c|c|c|c|c|c|}
\hline & $\begin{array}{l}1 \text { (Discordo } \\
\text { totalmente) }\end{array}$ & 2 & 3 & 4 & $\begin{array}{l}5 \text { (Concordo } \\
\text { totalmente) }\end{array}$ \\
\hline & $\begin{array}{c}n \\
(\%)\end{array}$ & $\begin{array}{c}n \\
(\%)\end{array}$ & $\begin{array}{c}n \\
(\%)\end{array}$ & $\begin{array}{c}n \\
(\%)\end{array}$ & $\begin{array}{c}n \\
(\%)\end{array}$ \\
\hline $\begin{array}{l}\text { Se um/a amigo/a quisesse doar gâmetas a } \\
\text { outro casal apoiaria a sua decisão }\end{array}$ & $\begin{array}{c}8 \\
(1,5 \%)\end{array}$ & $\begin{array}{c}13 \\
(2,5 \%)\end{array}$ & $\begin{array}{c}81 \\
(15,5 \%)\end{array}$ & $\begin{array}{c}148 \\
(28,4 \%)\end{array}$ & $\begin{array}{c}271 \\
(52,0 \%)\end{array}$ \\
\hline $\begin{array}{l}\text { Se um/a amigo/a quisesse receber gâmetas } \\
\text { apoiaria a sua decisão }\end{array}$ & $\begin{array}{c}6 \\
(1,2 \%)\end{array}$ & $\begin{array}{c}10 \\
(1,9 \%)\end{array}$ & $\begin{array}{c}72 \\
(13,8 \%)\end{array}$ & $\begin{array}{c}157 \\
(30,1 \%)\end{array}$ & $\begin{array}{c}276 \\
(53,0 \%)\end{array}$ \\
\hline $\begin{array}{l}\text { Se uma pessoa for estéril, a sua } 1 .^{a} \text { opção } \\
\text { deveria ser a adoção }\end{array}$ & $\begin{array}{c}103 \\
(19,8 \%)\end{array}$ & $\begin{array}{c}141 \\
(27,1 \%)\end{array}$ & $\begin{array}{c}186 \\
(35,7 \%)\end{array}$ & $\begin{array}{c}56 \\
(10,7 \%)\end{array}$ & $\begin{array}{c}35 \\
(6,7 \%)\end{array}$ \\
\hline $\begin{array}{l}\text { Se uma pessoa não pode ter filhos, então não } \\
\text { deveria tê-los }\end{array}$ & $\begin{array}{c}428 \\
(82,1 \%)\end{array}$ & $\begin{array}{c}43 \\
(8,3 \%)\end{array}$ & $\begin{array}{c}35 \\
(6,7 \%)\end{array}$ & $\begin{array}{c}8 \\
(1,5 \%)\end{array}$ & $\begin{array}{c}7 \\
(1,3 \%)\end{array}$ \\
\hline $\begin{array}{l}\text { A doação de gâmetas é uma boa forma de } \\
\text { ajudar casais que não podem ter filhos }\end{array}$ & $\begin{array}{c}7 \\
(1,3 \%)\end{array}$ & $\begin{array}{c}13 \\
(2,5 \%)\end{array}$ & $\begin{array}{c}91 \\
(17,5 \%)\end{array}$ & $\begin{array}{c}160 \\
(30,7 \%)\end{array}$ & $\begin{array}{c}250 \\
(48,0 \%)\end{array}$ \\
\hline
\end{tabular}

Dos 551 participantes, 318 (61,2\%) consideraram a possibilidade de doar gâmetas. Destes, 63,3\% ( $n=$ 202) dos indivíduos sentir-se-iam motivados por ajudar um casal que não pode ter filhos e $27,3 \%(n=87)$ sentir-se-ia a contribuir para ajudar o seu semelhante. Ainda em relação às motivações face à possibilidade de doar gâmetas, $41,7 \%(n=133)$ dos participantes indicaram não recear vir a ser contactados mais tarde e $49,5 \%(n=158)$ não se sentiriam incomodados por existir uma pessoa com a sua informação genética.

$\mathrm{Na}$ Tabela 3 são apresentados os resultados relativamente às atitudes dos participantes face à possibilidade de ter que recorrer a gâmetas de dador(es). Da amostra total de 551 indivíduos, 520 (94,4\%) sujeitos responderam afirmativamente, sendo que $31,9 \%(n=166)$ consideraram como totalmente verdadeiro para si que se sentiriam felizes por concretizar o sonho de virem a ser mãe/pai. De acrescentar que $38,1 \%$ ( $n=198)$ referiram ser totalmente verdadeiro para si sentirem-se contentes por cuidar de uma criança desde o nascimento. Ainda, $28,3 \%$ ( $n=147)$ referem que não se sentiriam nada receosos por virem a sentir que o filho não é realmente seu.

\section{Tabela 3}

Atitudes Face à Possibilidade de Ter Que Recorrer a Gâmetas de Dador(Es)

\begin{tabular}{|c|c|c|c|c|c|}
\hline & $\begin{array}{c}1 \text { (Nada } \\
\text { verdadeiro } \\
\text { para mim) }\end{array}$ & 2 & 3 & 4 & $\begin{array}{c}5 \text { (Totalmente } \\
\text { verdadeiro para } \\
\text { mim) }\end{array}$ \\
\hline $\begin{array}{l}\text { Feliz por concretizar o meu sonho de ser } \\
\text { mãe/pai }\end{array}$ & $\begin{array}{c}49 \\
(9,4 \%)\end{array}$ & $\begin{array}{c}39 \\
(7,5 \%)\end{array}$ & $\begin{array}{c}113 \\
(21,7 \%)\end{array}$ & $\begin{array}{c}153 \\
(29,4 \%)\end{array}$ & $\begin{array}{c}166 \\
(31,9 \%)\end{array}$ \\
\hline $\begin{array}{l}\text { Contente por cuidar de uma criança desde } \\
\text { o seu nascimento }\end{array}$ & $\begin{array}{c}40 \\
(7,7 \%)\end{array}$ & $\begin{array}{c}29 \\
(5,6 \%)\end{array}$ & $\begin{array}{c}80 \\
(15,4 \%)\end{array}$ & $\begin{array}{c}173 \\
(33,3 \%)\end{array}$ & $\begin{array}{c}198 \\
(38,1 \%)\end{array}$ \\
\hline $\begin{array}{l}\text { Feliz pelo facto de poder transmitir os } \\
\text { meus valores familiares }\end{array}$ & $\begin{array}{c}43 \\
(8,3 \%)\end{array}$ & $\begin{array}{c}27 \\
(5,2 \%)\end{array}$ & $\begin{array}{c}99 \\
(19,0 \%)\end{array}$ & $\begin{array}{c}164 \\
(31,5 \%)\end{array}$ & $\begin{array}{c}187 \\
(36,0 \%)\end{array}$ \\
\hline $\begin{array}{l}\text { Receoso/a por poder vir a sentir que o filho } \\
\text { não é realmente meu }\end{array}$ & $\begin{array}{c}147 \\
(28,3 \%)\end{array}$ & $\begin{array}{c}95 \\
(18,3 \%)\end{array}$ & $\begin{array}{c}112 \\
(21,5 \%)\end{array}$ & $\begin{array}{c}116 \\
(22,3 \%)\end{array}$ & $\begin{array}{c}50 \\
(9,6 \%)\end{array}$ \\
\hline $\begin{array}{l}\text { Apreensivo/a sobre eventuais problemas } \\
\text { de saúde genéticos }\end{array}$ & $\begin{array}{c}64 \\
(12,3 \%)\end{array}$ & $\begin{array}{c}91 \\
(17,5 \%)\end{array}$ & $\begin{array}{c}133 \\
(25,6 \%)\end{array}$ & $\begin{array}{c}145 \\
(27,9 \%)\end{array}$ & $\begin{array}{c}87 \\
(16,7 \%)\end{array}$ \\
\hline
\end{tabular}


No que se refere aos fatores que aumentariam/diminuiriam a probabilidade de doar gâmetas, verifica-se que para 39,2\% $(n=204)$ dos indivíduos receber uma compensação financeira não influenciaria a sua decisão de atuar como dador. Ter aconselhamento $(55,0 \% ; n=286)$, poder falar com outros dadores (45,0\%; $n=234)$ e ter mais informações sobre o que é não poder ter filhos (49,6\%; $n=258)$ revelam-se como os fatores importantes na decisão de doar gâmetas.

Tendo sido explorada a eventual existência de diferenças entre homens e mulheres relativamente às várias questões anteriormente abordadas, não foram apuradas quaisquer diferenças.

\section{Atitudes face à gestação de substituição}

Relativamente à GS, 73,5\% $(n=405)$ dos participantes revelam-se a favor da sua legalização em Portugal. Tanto no sexo masculino, como no feminino, a maioria dos sujeitos indica ser a favor da legalização da GS, não tendo sido encontradas diferenças entre os sexos. No entanto, a percentagem de homens que concorda com a legalização (76,5\%; $n=48)$ é superior à das mulheres $(72,7 \% ; n=154)$.

No que se refere a vir a usar esta técnica de reprodução medicamente assistida em caso de necessidade, $57,9 \%(n=300)$ dos indivíduos não colocaria essa hipótese. Novamente, não foram encontradas diferenças entre homens e mulheres relativamente a esta questão.

Analisando de forma mais específica as atitudes face à GS, a maioria dos participantes indica que se um/a amigo/a recorresse à GS apoiaria a sua decisão $(59,6 \% ; n=224)$ e apoiaria uma amiga se esta viesse a atuar como gestante de substituição (51,2\%; $n=189)$. Na globalidade, a maioria dos sujeitos (51,3\%; $n=$ 192) concorda totalmente que a GS é uma boa forma de ajudar casais com infertilidade.

Relativamente à possibilidade de recorrer à GS apenas 218, dos 551 participantes, considerariam essa hipótese. Como se pode observar na Tabela 4, constatou-se que 52,3\% ( $n=114)$ concorda totalmente que se sentiria contente por cuidar de uma criança desde o seu nascimento, 49,1\% ( $n=107)$ concorda totalmente que se sentiria feliz por concretizar o sonho de ser mãe/pai, 45,0\% ( $n=98)$ refere que seria totalmente verdadeiro para si sentir-se feliz pelo facto de poder transmitir os seus valores familiares e $38,1 \%(n=83)$ discorda totalmente que se sentiria receoso por poder vir a sentir que o filho não é realmente seu.

Por último, e no que concerne a apreensão sobre eventuais problemas que poderiam surgir durante a gravidez, os respondentes apresentam uma maior dispersão nas diferentes categorias de resposta, sendo a categoria 3 (ponto médio da escala de resposta) aquela em que se verifica uma maior frequência (33,5\%; $n=73)$. 


\section{Tabela 4}

Atitudes Face à Possibilidade de Recorrer à Gestação de Substituição

\begin{tabular}{|c|c|c|c|c|c|}
\hline & $\begin{array}{c}1 \text { (Nada } \\
\text { verdadeiro para } \\
\text { mim) }\end{array}$ & 2 & 3 & 4 & $\begin{array}{c}5 \text { (Totalmente } \\
\text { verdadeiro para } \\
\text { mim) }\end{array}$ \\
\hline $\begin{array}{l}\text { Feliz por concretizar o meu sonho de } \\
\text { ser mãe/pai }\end{array}$ & - & $\begin{array}{c}5 \\
(2,3 \%)\end{array}$ & $\begin{array}{c}35 \\
(16,1 \%)\end{array}$ & $\begin{array}{c}71 \\
(32,6 \%)\end{array}$ & $\begin{array}{c}107 \\
(49,1 \%)\end{array}$ \\
\hline $\begin{array}{l}\text { Contente por cuidar de uma criança } \\
\text { desde o seu nascimento }\end{array}$ & $\begin{array}{c}1 \\
(0,5 \%)\end{array}$ & $\begin{array}{c}2 \\
(0,9 \%)\end{array}$ & $\begin{array}{c}24 \\
(11,0 \%)\end{array}$ & $\begin{array}{c}77 \\
(35,3 \%)\end{array}$ & $\begin{array}{c}114 \\
(52,3 \%)\end{array}$ \\
\hline $\begin{array}{l}\text { Feliz pelo facto de poder transmitir os } \\
\text { meus valores familiares }\end{array}$ & $\begin{array}{c}3 \\
(1,4 \%)\end{array}$ & $\begin{array}{c}9 \\
(4,1 \%)\end{array}$ & $\begin{array}{c}35 \\
(16,1 \%)\end{array}$ & $\begin{array}{c}73 \\
(33,5 \%)\end{array}$ & $\begin{array}{c}98 \\
(45,0 \%)\end{array}$ \\
\hline $\begin{array}{l}\text { A certa altura, iria sentir que o filho } \\
\text { não era verdadeiramente meu }\end{array}$ & $\begin{array}{c}83 \\
(38,1 \%)\end{array}$ & $\begin{array}{c}48 \\
(22,0 \%)\end{array}$ & $\begin{array}{c}51 \\
(23,4 \%)\end{array}$ & $\begin{array}{c}23 \\
(10,6 \%)\end{array}$ & $\begin{array}{c}13 \\
(6,0 \%)\end{array}$ \\
\hline $\begin{array}{l}\text { Apreensivo/a sobre eventuais } \\
\text { problemas que poderiam surgir } \\
\text { durante a gravidez }\end{array}$ & $\begin{array}{c}19 \\
(8,7 \%)\end{array}$ & $\begin{array}{c}35 \\
(16,1 \%)\end{array}$ & $\begin{array}{c}73 \\
(33,5 \%)\end{array}$ & $\begin{array}{c}63 \\
(28,9 \%)\end{array}$ & $\begin{array}{c}28 \\
(12,8 \%)\end{array}$ \\
\hline
\end{tabular}

\section{Discussão}

O presente estudo pretendeu explorar as atitudes de indivíduos em idade reprodutiva relativamente a técnicas de reprodução medicamente assistida, especificamente em relação à doação de gâmetas e à gestação de substituição.

No que respeita às atitudes face à doação de gâmetas, foi possível verificar que, à semelhança do estudo de Provoost e colaboradores (2018), a maioria dos participantes revela uma atitude positiva perante a doação/receção de gâmetas. Ajudar um casal que não pode ter filhos e sentir-se a contribuir para o seu semelhante são os principais fatores referenciados como eventuais motivações dos participantes para virem a doar gâmetas (ovócitos/espermatozoides). Ainda neste sentido, outros fatores que se apresentam como relevantes para ponderarem vir a atuar como dadores de gâmetas são ter aconselhamento e dispor da oportunidade de falar com outros dadores. Atendendo a estes dados, poderá ser de utilidade, para os centros de reprodução medicamente assistida, nos quais se realiza a recolha de gâmetas, investir em ações de carácter informativo e disponibilizar serviços de aconselhamento com vista a um aumento do número de doações. Adicionalmente sessões de partilha de experiências de dadores, num formato presencial ou por recurso às tecnologias de informação (e.g., fóruns, websites) poderão, também, ser promovidas. Uma vez que os tratamentos no âmbito da medicina da reprodução têm vindo a aumentar na globalidade, mas também no que se refere a tratamentos realizados com gâmetas de dador (Nahata et al., 2017), é fundamental que as doações acompanhem este crescimento. No que concerne à receção de gâmetas, os resultados sugerem tratar-se de uma circunstância bem aceite pelos participantes. A maioria destes considerou que se sentiria feliz por concretizar o sonho da parentalidade e por cuidar de uma criança desde o seu nascimento, ainda que tivesse revelado alguma preocupação com a possibilidade da criança vir a evidenciar algum problema de saúde de natureza genética. De acrescentar que os resultados, no que respeita ao receio de vir a sentir que o filho não era verdadeiramente seu, sugerem uma maior valorização do vínculo afetivo comparativamente com a componente genética. Com efeito, apesar de 
alguma dispersão nas diferentes categorias de respostas, as percentagens foram mais expressivas nas categorias correspondentes à inexistência de preocupação com este aspeto. No entanto, é de referir que os participantes neste estudo não se encontravam numa circunstância efetiva de recorrer a tratamentos médicos com gâmetas doados. Com efeito, num estudo realizado com pessoas com um diagnóstico de infertilidade, constatou-se que estas revelavam uma clara preferência pela parentalidade genética, apresentando resistência no que respeita ao recurso a gâmetas de dador (Hendriks et al., 2017). Assim, estudos futuros poderão abordar as atitudes relativamente à receção de gâmetas em pessoas que possuam indicação para o uso de gâmetas de dador/a ou pessoas que tenham já beneficiado deste recurso. Relativamente às atitudes face à GS, a maioria dos indivíduos é a favor da sua legalização no nosso país. A este respeito, relembre-se que à altura da recolha dos dados esta era ainda uma questão pertinente, considerando que o enquadramento legal da época não permitia a realização desta técnica, havendo apenas propostas de lei em discussão. Independentemente de um posicionamento favorável em relação à legalização, na impossibilidade de ter filhos de forma espontânea e querendo ter um filho biológico, a maioria dos participantes não colocaria a hipótese de recorrer à GS. Com efeito, apesar de estudos conduzidos noutros países sugerirem que a GS não compromete o bem-estar, funcionamento conjugal e a capacidade de parentalidade do casal beneficiário (Gouveia, Galhardo, Cunha, \& Couto, 2017), bem como os indicadores perinatais das crianças nascidas por recurso a este método serem idênticos aos encontrados para outras técnicas de reprodução medicamente assistida (fertilização in vitro, injeção intracitoplasmática de espermatozoide e doação de ovócitos) (Söderström-Anttila et al., 2016), não existindo diferenças significativas em termos desenvolvimentais (Golombok, Blake, Casey, Roman, \& Jadva, 2013), a GS continua a gerar controvérsia, em virtude da sua complexidade. Apesar desta técnica não ser considerada como uma hipótese, em caso de necessidade, para a maioria dos sujeitos, os participantes que na impossibilidade de conceber um filho biológico recorreriam a esta técnica consideraram que se sentiriam felizes por concretizar o seu sonho de se tornar mãe/pai e que não se sentiriam receosos de vir a sentir que o filho não era realmente seu. De referir que, apesar das diferenças culturais e do facto da GS não ser permitida no Japão, num estudo realizado neste país, com 2500 sujeitos da população geral, com idades compreendidas entre os 20 e os 50 anos, também se observou uma maior percentagem de sujeitos (40,9\%) que eram favoráveis ao uso da GS, por comparação com $21,8 \%$ que discordavam da sua utilização (Yamamoto et al., 2018). Outro dado desta mesma investigação que vai ao encontro dos resultados obtidos no nosso estudo é o de as participantes do sexo feminino se revelarem mais conservadoras, ou seja, menos apoiantes do uso da GS, comparativamente com os participantes do sexo masculino (Yamamoto et al., 2018).

A presente investigação apresenta limitações metodológicas que devem ser ponderadas para estudos posteriores. A elevada escolarização da amostra e o elevado número de participantes do sexo feminino não viabiliza uma generalização dos resultados obtidos para a população geral. Para além disso, a inexistência de investigação em Portugal relativamente às atitudes perante a doação de gâmetas e a GS impossibilita, de igual forma, a comparação dos resultados obtidos.

Apesar das limitações acima elencadas, este estudo apresenta importantes contributos para a área. Reconhecendo que em Portugal estes tópicos não foram alvo de investigação no âmbito da psicologia, 
este trabalho revela-se pioneiro por abordar atitudes dos sujeitos em idade reprodutiva relativamente à doação/receção de gâmetas e à GS. Adicionalmente, é de referir que o tema abordado se reveste de atualidade e, neste sentido, poderá ser um contributo relevante para profissionais da área da fertilidade.

\section{Conclusão}

Na generalidade, os resultados obtidos neste trabalho remetem para a existência de atitudes favoráveis, de abertura e compreensão, por parte de indivíduos em idade reprodutiva, relativamente à utilização de gâmetas de dador em caso de necessidade para a concretização do seu protejo de parentalidade. Por sua vez, a possibilidade de atuar como dadores/as parece estar mais relacionada com a existência de aconselhamento e partilha da experiência com outros dadores, do que com a compensação financeira daí resultante, remetendo para uma motivação de natureza altruísta. Por último, os participantes evidenciaram uma atitude aberta e favorável relativamente à inclusão da GS no enquadramento da lei portuguesa, ainda que tenham evidenciado, por comparação com a doação de gâmetas, uma maior dispersão nas respostas manifestadas.

Conflito de interesses | Conflict of interest: nenhum | none.

Fontes de financiamento | Funding sources: nenhuma | none.

Contributos | Contributions: NC: Contributo significativo na recolha e tratamento dos dados, redação do manuscrito e análise estatística. AG: Contributo significativo no desenvolvimento do desenho metodológico, análise estatística e redação e revisão do manuscrito. MC: Contributo significativo na revisão do manuscrito.

\section{Referências}

Acórdão do Tribunal Constitucional n. ${ }^{\circ}$ 225/2018 de 7 de maio. Diário da República n. ${ }^{\circ}$ 87/2018, Série I [Portuguese legislation]. Lisboa: Tribunal Constitucional. Retrieved from https://data.dre.pt/eli/actconst/225/2018/05/07/p/dre/pt/html

Allan, H., \& Mounce, G. (2015). Managing infertility in primary care. Practice Nursing, 26(9), 440-443. https://doi.org/10.12968/pnur.2015.26.9.440

Boivin, J., Bunting, L., Collins, J. A., \& Nygren, K. G. (2007). International estimates of infertility prevalence and treatment-seeking: Potential need and demand for infertility medical care. Human Reproduction, 22(6), 1506-1512. https://doi.org/10.1093/humrep/dem046

Dar, S., Lazer, T., Swanson, S., Silverman, J., Wasser, C., Moskovtsev, ... Librach, C. L. (2015). Assisted reproduction involving gestational surrogacy: An analysis of the medical, psychosocial and legal issues: experience from a large surrogacy program. Human Reproduction, 30(2), 345-352. https://doi.org/10.1093/humrep/deu333

De Geyter, C., Calhaz-Jorge, C., Kupka, M. S., Wyns, C., Mocanu, E., Motrenko, T., ... Goossens, V. (2018). ART in Europe, 2014: Results generated from European registries by ESHRE. Human Reproduction, 33(9), 15861601. https://doi.org/10.1093/humrep/dey242

Decreto-Lei n. ${ }^{\circ}$ 32/2006 de 26 de julho. Diário da República n. ${ }^{\circ}$ 143/2006, Série / [Portuguese legislation]. Lisboa: Assembleia da República. Retrieved from https://data.dre.pt/eli/lei/32/2006/07/26/p/dre/pt/html

ESHRE Task Force on Ethics and Law. (2002). Gamete and embryo donation. Human Reproduction, 175), 1407-1408. Retrieved from https://www.eshre.eu/Specialty-groups/Special-Interest-Groups/Ethics-andLaw/Documents-of-the-Task-Force-Ethics-Law 
Goldfarb, J. M., Austin, C., Peskin, B., Lisbona, H., Desai, N., \& de Mola, J. R. L. (2000). Fifteen years experience with an in-vitro fertilization surrogate gestational pregnancy programme. Human Reproduction, 15(5), 1075-1078. https://doi.org/10.1093/humrep/15.5.1075

Golombok, S., Blake, L., Casey, P., Roman, G., \& Jadva, V. (2013). Children born through reproductive donation: A longitudinal study of psychological adjustment. Journal of Child Psychology and Psychiatry, 54(6), 653660. https://doi.org/10.1111/jcpp.12015

Gouveia, J., Galhardo, A., Cunha, M., \& Couto, M. (2017). Gestação de substituição: aspetos psicológicos - Uma revisão da literatura [Surrogacy for infertility: Psychological aspects - A review of the literature]. Psicologia, Saúde \& Doenças, 18(1), 248-262. http://doi.org/10.15309/17psd180120

Hendriks, S., Peeraer, K., Bos, H., Repping, S., \& Dancet, E. A. F. (2017). The importance of genetic parenthood for infertile men and women. Human Reproduction, 32(10), 2076-2087. https://doi.org/10.1093/humrep/dex256

Lampic, C., Svanberg, A. S., Karlström, P., \& Tydén, T. (2006). Fertility awareness, intentions concerning childbearing and attitudes towards parenthood among female and male academics. Human Reproduction, 21(2), 558-564. https://doi.org/10.1093/humrep/dei367

Lei n. ${ }^{\circ}$ 25/2016 de 22 de agosto. Diário da República n. ${ }^{\circ}$ 160/2016, Série / [Portuguese legislation]. Lisboa: Assembleia da República. Retrieved from https://data.dre.pt/eli/lei/25/2016/08/22/p/dre/pt/html

Marôco, J. (2010). Análise estatística como PASW Statistics (ex-SPSS)[Statistical analysis with PASW Statistics (ex-SPSS)]. Pêro Pinheiro: ReportNumber.

Nahata, L., Stanley, N., \& Quinn, G. (2017). Gamete donation: Current practices, public opinion, and unanswered questions. Fertility and Sterility, 1076), 1298-1299. https://doi.org/10.1016/j.fertnstert.2017.04.001

Nouri, K., Huber, D., Walch, K., Promberger, R., Buerkle, B., Ott, J., \& Tempfer, C. B. (2014). Fertility awareness among medical and non-medical students: A case-control study. Reproductive Biology and Endocrinology, 12(1), 1-9. https://doi.org/10.1186/1477-7827-12-94

Pennings, G., Ravel, C., Girard, J. M., Domin-Bernhard, M., \& Provoost, V. (2018). Attitude towards reciprocity as a motive for oocyte donation. European Journal of Obstetrics \& Gynecology and Reproductive Biology, 225, 194-198. https://doi.org/10.1016/j.ejogrb.2018.04.038

Provoost, V., Van Rompuy, F., \& Pennings, G. (2018). Non-donors' attitudes towards sperm donation and their willingness to donate. Journal of Assisted Reproduction and Genetics, 35(1), 107-118. https://doi.org/10.1007/s10815-017-1036-x

Rovei, V., Gennarelli, G., Lantieri, T., Casano, S., Revelli, A., \& Massobrio., M. (2010). Family planning, fertility awareness and knowledge about Italian legislation on assisted reproduction among Italian academic students. Reproductive Biomedicine Online, 2077), 873-879. https://doi.org/10.1016/j.rbmo.2010.03.024

Salama, M., Isachenko, V., Isachenko, E., Rahimi, G., Mallmann, P., Westphal, L. M., ... \& Patrizio, P. (2018). Cross border reproductive care (CBRC): A growing global phenomenon with multidimensional implications (a systematic and critical review). Journal of Assisted Reproduction and Genetics, 35(7), 1277-1288. https://doi.org/10.1007/s10815-018-1181-x

Silva-Carvalho, J. L., \& Santos, A. (2009). Estudo Afrodite: Caracterização da infertilidade em Portugal - Estudo na comunidade [Aphrodite Study: Characterization of infertility in Portugal - Study in the community] (Vol. 1, pp. 9-73). Porto: Faculdade de Medicina da Universidade do Porto.

Söderström-Anttila, V., Wennerholm, U.-B., Loft, A., Pinborg, A., Aittomäki, K., Romundstad, L. B., \& Bergh, C. (2016). Surrogacy: Outcomes for surrogate mothers, children and the resulting families - A systematic review. Human Reproduction Update, 22(2), 260-276. https://doi.org/10.1093/humupd/dmv046

Yamamoto, N., Hirata, T., Izumi, G., Nakazawa, A., Fukuda, S., Neriishi, K., ... Osuga, Y. (2018). A survey of public attitudes towards third-party reproduction in Japan in 2014. PLOS ONE, 13(10), e0198499. https://doi.org/10.1371/journal.pone.0198499

Zegers-Hochschild, F., Adamson, G. D., Dyer, S., Racowsky, C., de Mouzon, J., Sokol, R., ... van der Poel, S. (2017). The international glossary on infertility and fertility care. Fertility and Sterility, 108(3), 393-406. https://doi.org/10.1016/j.fertnstert.2017.06.005 